University of Wollongong

Research Online

Faculty of Engineering and Information

Faculty of Engineering and Information

Sciences - Papers: Part A

Sciences

$1-1-2014$

\title{
Time spent on daytime direct care activities by personal carers in two Australian residential aged care facilities: a time-motion study
}

Siyu Qian

University of Wollongong, sq992@uowmail.edu.au

Ping Yu

University of Wollongong, ping@uow.edu.au

David M. Hailey

University of Wollongong, dhailey@uow.edu.au

Zhenyu Zhang

University of Wollongong, zhenyu@uow.edu.au

Pam Davy

University of Wollongong, pjd@uow.edu.au

See next page for additional authors

Follow this and additional works at: https://ro.uow.edu.au/eispapers

Part of the Engineering Commons, and the Science and Technology Studies Commons

Research Online is the open access institutional repository for the University of Wollongong. For further information contact the UOW Library: research-pubs@uow.edu.au 


\title{
Time spent on daytime direct care activities by personal carers in two Australian residential aged care facilities: a time-motion study
}

\begin{abstract}
Objective. To examine the time, frequency and duration of each direct care activity conducted by personal carers in Australian residential aged care homes. Methods. A time-motion study was conducted to observe 46 personal carers at two high-care houses in two facilities (14 days at Site 1 and 16 days at Site 2). Twenty-three direct care activities were classified into eight categories for analysis. Results. Overall, a personal carer spent approximately $45 \%$ of their time on direct care, corresponding to $3.5 \mathrm{~h}$ in an 8 -h daytime shift. The two sites had similar ratios of personal carers to residents, and each resident received 30 min of direct care. No significant differences between the two sites were found in the time spent on oral communication, personal hygiene and continence activities. Personal carers at Site 1 spent significantly less time on toileting and mobility activities than those at Site 2, but more time on lunch activity. Although oral communication took the longest time $(2 \mathrm{~h})$, it occurred concurrently with other activities (e.g. dressing) for $1.5 \mathrm{~h}$. Conclusions. The findings provide information that may assist decision makers in managing the operation of highcare residential aged care facilities, such as planning for task allocation and staffing.
\end{abstract}

\section{Keywords}

carers, personal, activities, study, care, time, direct, daytime, spent, motion, facilities, aged, residential, australian, two

\section{Disciplines \\ Engineering | Science and Technology Studies}

\section{Publication Details}

Qian, S., Yu, P., Hailey, D. M., Zheng, Z., Davy, P. J. \& Nelson, M. I. (2014). Time spent on daytime direct care activities by personal carers in two Australian residential aged care facilities: a time-motion study. Australian Health Review, 38 (2), 230-237.

\section{Authors}

Siyu Qian, Ping Yu, David M. Hailey, Zhenyu Zhang, Pam Davy, and Mark I. Nelson 


\title{
Time spent on daytime direct care activities by personal carers in two Australian residential aged care facilities: a time- motion study
}

\section{(Pre-publication version)}

\author{
Siyu Qian ${ }^{1}$, Ping Yu ${ }^{1,3}$, David M. Hailey ${ }^{1}$, Zhenyu Zhang ${ }^{1}$, Pamela J. Davy ${ }^{2}$, Mark I. Nelson ${ }^{2}$ \\ ${ }^{1}$ School of Information Systems and Technology, University of Wollongong, Northfields Avenue, \\ Wollongong, NSW 2522, Australia. \\ ${ }^{2}$ School of Mathematics and Applied Statistics, University of Wollongong, Northfields Avenue, \\ Wollongong, NSW 2522, Australia. \\ ${ }^{3}$ Corresponding author: Email: ping@uow.edu.au
}

\begin{abstract}
Objective. To examine the time, frequency and duration of each direct care activity conducted by personal carers in Australian residential aged care homes.

Methods. A time-motion study was conducted to observe 46 personal carers at two highcare houses in two facilities (14 days at Site 1 and 16 days at Site 2). Twenty-three direct care activities were classified into eight categories for analysis.

Results. Overall, a personal carer spent approximately $45 \%$ of their time on direct care, corresponding to $3.5 \mathrm{~h}$ in an 8-h daytime shift. The two sites had similar ratios of personal carers to residents, and each resident received $30 \mathrm{~min}$ of direct care. No significant differences between the two sites were found in the time spent on oral communication, personal hygiene and continence activities. Personal carers at Site 1 spent significantly less time on toileting and mobility activities than those at Site 2, but more time on lunch activity. Although oral communication took the longest time ( $2 \mathrm{~h})$, it occurred concurrently with other activities (e.g. dressing) for $1.5 \mathrm{~h}$.

Conclusions. The findings provide information that may assist decision makers in managing the operation of high-care residential aged care facilities, such as planning for task allocation and staffing.
\end{abstract}

Key words: activity, long-term care, nursing home, observation, personal care.

What is known about the topic? Overall, $30 \%-45 \%$ of the care staff's time is spent on direct care in residential aged care facilities.

What does this paper add? This paper adds knowledge about how much time is required to conduct each direct care activity and the frequency and duration of 
conducting these activities to meet residents' day-to-day care needs in two high-care houses in two aged care facilities.

What are the implications for practitioners? On average, a resident with high-care needs requires 30 min direct care. There may exist a basic minimum desirable ratio of personal carers to residents in high-care facilities. Residents' toileting needs are high after meals. Communication with residents represents an essential role in providing care.

\section{Introduction}

With a rapidly aging population, the number of older people needing residential aged care in Australia is expected to increase. ${ }^{1}$ However, the direct care workforce has a chronic shortage of trained staff. ${ }^{2}$ This represents a big challenge for residential aged care facilities (RACFs) to provide quality, safe, efficient and appropriate delivery of care. Personal carers (PCs) provide personal care to meet aged care residents' daily needs (e.g. personal hygiene). Richardson and Martin, in 'The Care of Older Australians: A Picture of Residential Aged Care Workforce', reported that more than $66 \%$ of PCs felt that they did not have enough time to spend with each resident. ${ }^{3}$ This leads to a question about how PCs spent their time with residents.

Knowledge about how PCs spent their time can improve nursing managers' understanding about the time required to directly meet residents' care needs, thus estimating staff workload. This can inform evidence-based decisions on whether task reallocation is needed ${ }^{4}$ in order to maintain quality of care and improve work efficiency. ${ }^{5}$

In addition, this knowledge will set the baseline for examining the impact of any implemented change, ${ }^{4}$ such as introduction of an electronic information system. ${ }^{6-8}$ Furthermore, such details are important in informing public policy or managerial decision on staffing levels, ${ }^{9}$ either for newly built aged care facilities or existing facilities in which resident case mix has changed.

In lean management, time is also an important measure. ${ }^{10}$ Lean management aims to use less to do more. ${ }^{11}$ By making the performance visible, reducing the number of non-valueadding activities, eliminating waste and standardising the work, lean management has the potential to optimise care processes. ${ }^{10-12}$ The first step to lean management is making the care process visible, and time is a key measure of the process. ${ }^{12}$

Direct care activities are those directly involved with residents. ${ }^{13-15}$ Previous studies have focused on the time spent on caring for dementia residents. ${ }^{16,17}$ Several studies provided broad understanding of how PCs spend their time. They reported that $30 \%-45 \%$ of a PC's time was spent on direct care in RACFs. ${ }^{6,15,18}$ However, none of these studies 
reported how much time was allocated to each direct care activity in high-care RACFs. This limits the understanding about the time needed for meeting different care needs.

To fill this gap, the aim of the present study was to examine how much time is spent on each direct care activity, and the frequency and duration of conducting these activities.

\section{Methods}

A time-motion observational study was conducted in 2010. An observer shadowed a participant and recorded this person's activities sequentially for a period of time using a predefined classification system of activities. ${ }^{19}$

\section{Classification of activities}

The classification system was developed over the course of three focus group discussions. Each focus group contained three researchers and three registered nurses (RNs) with extensive work experience in aged care. A previously validated classification system ${ }^{14}$ was given to the focus group as an initial version. During the three discussions, this system was tuned to make it more appropriate for a time-motion study. The final version contained 58 activities that were grouped into direct care, indirect care, infection control, oral communication, documentation, transit, staff break and other activities not included in the previous categories. ${ }^{15}$

Only direct care activities are presented in this paper. These were further classified into eight subcategories, which were intended to provide adequate granularity, yet remaining abstract enough for conceptualisation and presentation (see Table1). To name each category, the wording from the aged care funding instrument was used.

\section{Settings}

Two RACFs were recruited. The first, operated by a non-profit organisation, was located in Sydney and had 155 beds. The second was a stand-alone non-profit facility in Newcastle with 108 beds. Both RACFs provided low and high care. Older people living in a low-care house require limited help with their activities of daily living, whereas those living in a high-care house fully depend on care staff. $^{20}$

In Australia, $60 \%$ of the RACFs are not-for-profit, with $45 \%$ of these having more than 60 beds. $^{21}$ Of the large facilities that operate more than 60 beds, nearly $60 \%$ provide both low and high care. ${ }^{2}$ The RACFs evaluated in the present study fell into this category of facilities.

Observations were conducted in two high-care houses of the two study RACFs. Site 1 had 32 residents. One RN and 4.5 fulltime equivalent PCs worked in a daytime shift. Site 2 had 23 residents who were looked after by one RN and three full-time PCs. 


\section{Ethics approval}

Ethics approval was acquired from the University of Wollongong ethics review board upon agreement from the participant RACFs. Because only PCs were observed, consent was not sought from residents. When a PC was providing care to a resident in a bathroom or behind a privacy curtain, the observer stayed outside. The activities performed by the PC were identified by listening to the care instructions the PC gave to the resident.

\section{Training of the observer}

Observation was conducted by a single observer to maintain the consistency of recording. A 6-h orientation on observation practice was provided to the observer by a researcher experienced in observational studies in RACFs. Both independently observed four PCs in one shift at Site 1 . More than $95 \%$ agreement was achieved in their recorded data, with differences in the remaining $5 \%$ being resolved by discussion and consensus. According to the suggestion of Pelletier and Duffield, ${ }^{22}$ the observer had adequate competence to collect valid and reliable data.

\section{Selection of participants}

The observer randomly approached a PC, explained the purpose and procedure of the study and asked for the person's written consent. Once the person gave written consent, the observation started. If consent was not given, the observer approached another PC. Where possible, a different PC was observed the next day to maximise the number of participants.

\section{Data collection}

The observer was introduced to the PCs and RNs by the nursing manager 1 day before the formal observation. Because of resource constraint, observation time was confined to daytime shift (morning and part of the afternoon shift). Once the observation started, no direct communication was made between the observer and the PC.

At Site 1, 11 PCs were observed over 14 days. Three were observed twice. On each day, one PC was continuously observed from 7 am to either 2 or $3 \mathrm{pm}$. At Site 2, 27 PCs were observed from 10 am to 5 pm over 16 days, with five observed twice. Two PCs were observed each day.

Activities and their start time were recorded sequentially in an Excel spreadsheet using a tablet computer. The start time of an activity was the end time of its precedent activity. During the first 7 days at Site 1, the observer noticed that when a PC spoke with a resident, the person could simultaneously conduct another activity. This communication was identified as 'concurrent oral communication', whereas communication that happened on its own was recorded as 'pure oral communication'. To ensure an accurate 
recording of the time spent on oral communication, the end time of each oral communication was recorded for the remaining 7 days at Site 1 and for all 16 days at Site 2 .

\section{Results}

Overall, 45.4\%of the PCs' time was spent on direct care activities, corresponding to $3.5 \mathrm{~h}$ over an 8-h daytime shift. At Site 1, 1943 direct care instances were recorded, with an average of 24 per h, compared with 2913 at Site 2, with an average of 32 per h.

Time spent with each resident

Similar PC : resident ratios were found at the two sites $(1: 7.1$ at Site 1 and $1: 7.7$ at Site 2). Each resident received a similar amount of direct care time (27 $\mathrm{min}$ at Site 1 and 29 min at Site 2) in an 8-h shift.

\section{Comparison of activity time}

No significant difference between the two sites was found in the overall time that a PC spent on direct care (Table2). At both sites, a PC spent most time on oral communication ( $2 \mathrm{~h}$ ), followed by personal hygiene (43 $\mathrm{min}$ ). No significant differences between sites were found in communication and personal hygiene, or with continence activities.

Significant differences between the two sites were found in toileting, mobility and lunch activities. A PC at Site 1 spent less time on toileting (7 vs $17 \mathrm{~min}$ for Site 1 vs Site 2, respectively; $\mathrm{P}<0.001$ ) and mobility activities (34 vs $53 \mathrm{~min}$ for Site 1 vs Site 2, respectively; $\mathrm{P}<0.001$ ) than a $\mathrm{PC}$ at Site 2 , but more time on lunch activities (26 vs $7 \mathrm{~min}$ for Site 1 vs Site 2, respectively; $\mathrm{P}<0.001$ ).

\section{Comparison of activity duration}

With regard to the duration of an activity (Table2), a significant difference between sites was found only for communication with a resident (91 vs 72 s for Site 1 vs Site 2, respectively; $\mathrm{P}=0.04)$.

\section{Concurrent communication with a resident}

Of the three types of oral communication activities (Table1), communication with a resident was the most time consuming. It took approximately $2 \mathrm{~h}$ in an 8 -h shift, with 30 min spent on pure oral communication and $1.5 \mathrm{~h}$ on concurrent oral communication (Table3). The time spent on conducting direct care activities while a PC was talking to a resident was $54 \mathrm{~min}$. This was less than the total time spent on concurrent oral communication, because a PC may be concurrently conducting an indirect care activity. 
Figure1shows the percentage of instances of direct care activities for each hour over the observation period. No significant difference was found between the two sites in the hours between 11 am and $2 \mathrm{pm}$, when $35.5 \%$ of activity instances occurred.

Figure2 shows the distribution of each direct care activity over the observation hours. For example, at Site 1 toileting activity occurred more frequently after breakfast (9-10 am; 16\% of the toileting activities) and lunch (1-2 pm; 37\%) than the other hours. At Site 2, toileting activity also peaked after meal time.

\section{Discussion}

In an RACF, some of a resident's daily care needs, such as meals, must be met on a regular schedule. Some care needs, such as recreational activity, can be delivered at a relatively flexible time interval. Other activities may happen randomly depending on the health condition and psychological and physical well being of the person on the day. These randomly timed activities make it a challenge to predict what the next care activity will be and how much time is required to complete it. This has increased the challenge for the managers in RACFs to adequately plan and deliver care services that meet older people's needs.

This study provides information relevant to the patterns of direct care activities that may be of help to managers and other staff in RACFs. Because the two study RACFs were completely independent of each other in their organisation of care services, facility layout and care staff, some common findings at both facilities may be applicable to other highcare services in Australian residential aged care.

\section{Common findings at both RACFs}

The time recorded in this study was that spent on directly interacting with a resident rather than the full time needed to complete a direct care task, which may involve other activities, such as indirect care. For example, to complete a shower task, a PC needs to first complete certain indirect care activities, such as preparing towels.

The common findings at both sites include: (1) no significant differences in the overall time spent on direct care; (2) the PC : resident ratio was approximately one PC to seven or eight residents in a high-care RACF, and this may indicate that there could be a basic, common staff ratio that was followed by service providers to run a high-care facility; (3) on average, each resident received $30 \mathrm{~min}$ direct care in an 8-h day shift; (4) no significant differences in the time spent on oral communication, personal hygiene and continence activities; (5) no significant differences in the percentage of direct care activities that occurred between 11 am and $2 \mathrm{pm}$, which may suggest that residents' needs over this period of time were similar; and (6) the high occurrence of toileting activities 
after meal time at both sites may indicate that residents' toileting needs were high after meals.

Previous studies ${ }^{6,15,18}$ only presented the total direct care time, which was consistent with the first point presented above, but did not address the other points. The present study has advanced knowledge by providing more detailed information about each direct care activity. Nursing managers may use this knowledge to estimate staff workload, make decisions on task re-allocation ${ }^{4}$ and staffing levels, ${ }^{9}$ evaluate the impact of implemented changes ${ }^{4}$ and develop strategies to optimise care processes.

These findings were drawn from the observational study conducted in two RACFs and contextual factors should be considered when referencing the findings. The generalisability of the findings should be validated by a larger-scale investigation to inform public policy on staffing levels.

\section{Differences in toileting and mobility activities}

Personal carers at Site 1 spent significantly less time on toileting and mobility activities than their counterparts at Site 2 and conducted these two activities less frequently. This may be due to the difference in functional dependence levels of the residents. Another possible reason could be the different toileting practices: a ceiling hoist system was used at Site 2, but not at Site 1 .

Assistive technologies may reduce physical burden on $\mathrm{PCs}^{23}$ and the convenience of using them may motivate PCs to deliver toileting care more often to maintain residents' continence. However, if not used only when necessary, it has the potential to reduce the frequency with which residents are assisted to walk. In turn, this may lead to dramatic loss of a resident's walking ability, which is a serious problem.

\section{Communication with residents}

Similar to what was found in a previous study, ${ }^{6}$ oral communication was often conducted concurrently with direct care activities. An oral communication could be instrumental or social. It was likely that, while conducting a direct care activity, a PC spoke with the resident to instruct the person in a care task so as to acquire cooperation ${ }^{6,15}$ or for the purpose of social engagement.

Communication with residents is essential for increasing familiarity and maintaining personal relationships with residents, and these contribute to the maintenance of residents' sense of competence and dignity. ${ }^{24}$ Future research may examine how much social communication was engaged in while completing an instrumental task and how much occurred outside this task. This is important in the context of promoting person-centred care. 


\section{Limitations}

The results of the present study may not be representative of aged care facilities nationally because the sites were not selected on this basis. The exclusion of some of the communication data at Site 1 is also a limitation of the present study.

There was a potential for PCs to change work behaviour under constant observation due to the Hawthorne effect. ${ }^{25}$ Several previous studies in healthcare settings, including RACFs, have suggested that the Hawthorne effect is not significant in direct observation; ${ }^{6,26,27}$ consequently, we did not measure it in the present study.

Data about the functional dependence levels of residents, such as their ability to feed themselves, were not collected, but the managers at both sites suggested that their residents need high care.

Because the observer did not directly observe a PC when they were providing care in a bathroom or behind a privacy curtain, there could be inaccuracy in recording activities when a PC was socialising with a resident. In this situation, the activity was indicated by the activity that was performed initially. There was no way to examine how much difference was generated by this practice under the ethics condition of the present study.

It was possible that the time recorded on oral communication was increased by the effort made by a PC to allow the observer to hear the care instructions. Because the care instructions were necessary in provision of care and no direct communication was made between the observer and the PC, it was assumed that there was minimal impact on communication time.

\section{Conclusions}

We conducted a time-motion study in two high-care houses in two Australian RACFs to examine the time, frequency and duration of 23 direct care activities that meet older people's day-to-day care needs. At both sites, a PC spent $3.5 \mathrm{~h}$ on direct care in an 8-h shift, with $30 \mathrm{~min}$ for each resident. The PC : resident ratios were similar at the two sites (one PC looked after seven to eight residents). No significant differences were found between the two sites in the time spent on oral communication, personal hygiene and continence activities. Personal carers at Site 1 spent less time on toileting and mobility activities, but more time on lunch than their counterparts at Site 2. The pattern of occurrence of each care activity in different hours was also investigated.

Further investigation about the care process, such as identifying non-value-adding activities, examining equipment changeover time and resident's waiting time before needs are met, is needed to improve process ability to meet residents' needs.

\section{References}


1. Productivity Commission. Economic implications of an ageing Australia. Canberra: Productivity Commission; 2005.

2. Martin B, King D. Who cares for older Australians? A picture of the residential and community based aged care workforce, 2007. Canberra: Commonwealth of Australia; 2008.

3. Richardson S, Martin B. The care of older Australians: a picture of the residential aged care workforce. Adelaide: The National Institute of Labour Studies, Flinders University; 2004.

4. Urden LD, Roode JI. Work sampling. A decision-making tool for determining resources and work redesign. $J$ Nurs Adm 1997; 27(9): 34-41. doi:10.1097/00005110-199709000-00009

5. Thomson MS, Gruneir A, Lee M, Baril J, Field TS, Gurwitz JH, et al. Nursing time devoted to medication administration in long-term care: clinical, safety, and resource implications. $J$ Am Geriatr Soc 2009; 57(2): 266-72. doi:10.1111/j.15325415.2008.02101.x

6. Munyisia EN, Yu P, Hailey D. How nursing staff spend their time on activities in a nursing home: an observational study. $J$ Adv Nurs 2011; 67(9): 1908-17. doi:10.1111/j.1365-2648.2011.05633.x

7. Cornell P, Herrin-Griffith D, Keim C, Petschonek S, Sanders AM, D’Mello S,et al. Transforming nursing workflow, part 1: The chaotic nature of nurse activities. J Nurs Adm 2010; 40(9): 366-73. doi:10.1097/NNA.0b013e3181ee4261

8. Poulos CJ, Gazibarich BM, Eagar K. Supporting work practices, improving patient flow and monitoring performance using a clinical information management system. Aust Health Rev 2007; 31(5): 79-85. doi:10.1071/AH070S79

9. Abbey M, Chaboyer W, Mitchell M. Understanding the work of intensive care nurses: a time and motion study. Aust Crit Care 2012; 25(1): 13-22. doi:10.1016/j.aucc.2011.08.002

10. Anupindi R, Chopra S, Deshmukh SD, Van Mieghem JA, Zemel E. Managing business process flows. Upper Saddle River, NJ: Pearson/Prentice Hall; 2011.

11. Womack JP, Miller D. Going lean in health care. Cambridge, MA: Institute for Healthcare Improvement; 2005.

12. Kim CS, Spahlinger DA, Kin JM, Billi JE. Lean health care: what can hospitals learn from a world-class automaker? J Hosp Med 2006; 1(3): 191-9. doi:10.1002/jhm.68

13. Westbrook JI, Ampt A. Design, application and testing of the work observation method by activity timing (wombat) to measure clinicians' patterns of work and communication. Int $J$ Med Inform 2009; 78: S25-33. doi:10.1016/j.ijmedinf.2008.09.003

14. Munyisia EN, Yu P, Hailey D. Development and testing of a work measurement tool to assess caregivers' activities in residential aged care facilities. Stud Health Technol Inform 2010; 160: 1226-30. doi:10.3233/978-1-60750-588-4-1226 
15. Qian SY, Yu P, Zhang ZY, Hailey D, Davy P, Nelson M. The work pattern of personal care workers in two Australian nursing homes: a time-motion study. BMC Health Serv Res 2012; 12(1): 305. doi:10.1186/1472-6963-12-305

16. Luttenberger K, Graessel E. Recording care time in nursing homes: development and validation of the 'RUD-FOCA' (resource utilization in dementia-formal care). Int Psychogeriatr 2010; 22: 1291-300. doi:10.1017/S1041610210000487

17. Kobayashi N, Yamamoto M. Impact of the stage of dementia on the time required for bathing-related care: a pilot study in a Japanese nursing home. Int J Nurs Stud 2004; 41(7): 767-74. doi:10.1016/j.ijnurstu.2004.03.003

18. Thorsell K, Nordström B, Fagerström L, Sivberg B. Time in care for older people living in nursing homes. Nursing Research and Practice 2010; 2010: 148435. doi:10.1155/2010/148435

19. Gilbreth FB. Motion study a method for increasing the efficiency of the workman. London: Constable and Company; 1911.

20. Productivity Commission. Trends in aged care services. Canberra: Productivity Commision; 2008.

21. Australian Institute of Health and Welfare (AIHW). Residential aged care in Australia 2010-11: a statistical overview. Canberra: AIHW; 2012.

22. Pelletier D, Duffield C. Work sampling: valuable methodology to define nursing practice patterns. Nurs Health Sci 2003; 5(1): 31-8. doi:10.1046/j.14422018.2003.00132.x

23. Productivity Commission. Caring for older Australians. Canberra: Commonwealth of Australia: Canberra; 2011.

24. Bowers BJ, Esmond S, Jacobson N. The relationship between staffing and quality in long-term care facilities: exploring the views of nurse aides. J Nurs Care Qual 2000; 14(4): 55-64. doi:10.1097/00001786-200007000-00009

25. Finkler SA, Knickman JR, Hendrickson G, Lipkin MJ, Thompson WG. A comparison of work-sampling and time-and-motion techniques for studies in health services research. Health Serv Res 1993; 28(5): 577-97.

26. Ampt A, Westbrook J, Creswick N, Mallock N. A comparison of self-reported and observational work sampling techniques for measuring time in nursing tasks. $J$ Health Serv Res Policy 2007; 12(1): 18-24. doi:10.1258/135581907779497576

27. Schnelle JF, Ouslander JG, Simmons SF. Direct observations of nursing home care quality: does care change when observed? J Am Med Dir Assoc 2006; 7(9): 541-4. doi:10.1016/j.jamda.2006.03.009 
Table 1. Classification of personal carers' direct care activities

\begin{tabular}{lll}
\hline Direct care categories & & Activities \\
\hline Assessment & 1 & Physical assessment \\
& 2 & Specimen collection; urine collection \\
Personal hygiene & 3 & Weighing a resident \\
& 4 & Routine hygiene (e.g. daily shower or wash) \\
& 5 & Continence related hygiene (e.g. shower or wash following pad change) \\
& 7 & Oral care \\
Toileting & 8 & Shave or grooming \\
& 9 & Assisting a resident with hand washing following the use of the toilet \\
Continence & 10 & Toileting (prompted by a resident) \\
& 11 & Toileting (prompted by a personal carer) \\
Mobility & 12 & Scheduled toileting \\
& 13 & Pad check \\
Medication & 14 & Pad change \\
Nutrition & 15 & Resident mobility; passive and active exercises; turning a resident in bed \\
& 16 & Assisting a resident with transfer to and from a bed, a chair etc. \\
Oral communication & 17 & Transferring a resident to or from the dining room or boardroom \\
& 18 & Medication administration \\
& 19 & Assisting a resident with eating and drinking (include feeding systems) \\
& 20 & Assisting a resident with food (e.g. cutting up, uncovering or delivery of food) \\
& 22 & Assisting a resident to receive a phone call \\
& 23 & Attending to a resident call for assistance \\
& & Communication with a resident \\
\hline
\end{tabular}


Table 2. Time, frequency and duration of each direct care activity at the two sites (observation times: 7 am to 2 or $3 \mathrm{pm}$ at Site 1; 10 am to $5 \mathrm{pm}$ at Site 2) Unless indicated otherwise, data show the mean \pm s.d., with $95 \%$ confidence intervals in parentheses. Data regarding communication with a resident during the first 7 days at Site 1 were excluded from analysis because the end time was not recorded and concurrent communication could not be identified. The percentage of time spent on assessment and medication at the two sites was not compared because the sample sizes were not large enough for statistical comparison. The total time spent on nutrition was not compared owing to different observation time periods at the two sites, but lunch time was compared and is presented in the text. Asterisks indicate significant differences between the two sites in the percentage of time spent on an activity or in the duration of conducting an activity

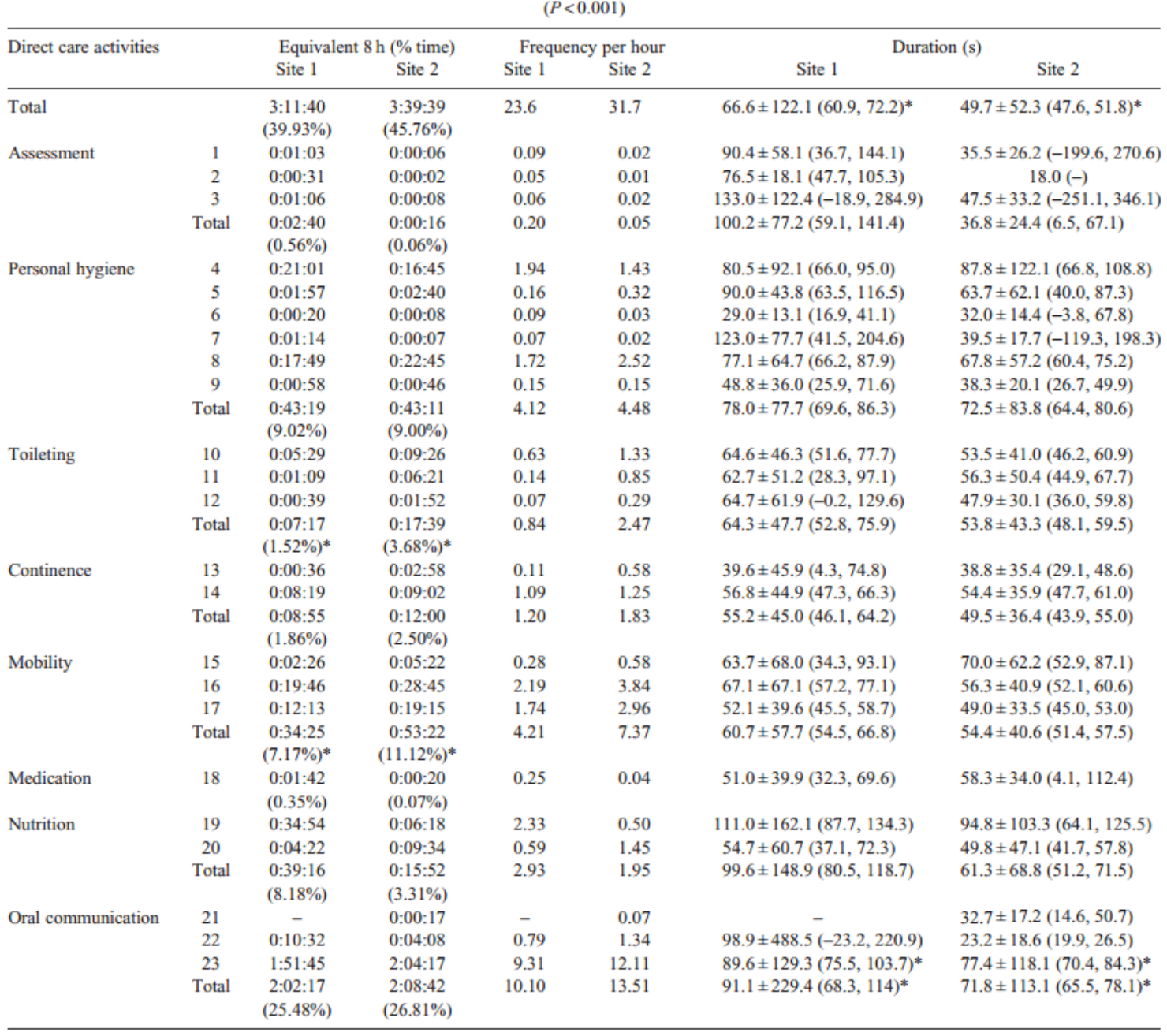


Table 3. Direct care activities that happened concurrently with communication with a resident, combing the data collected at the two sites after the first 7 days of observation

\begin{tabular}{lll}
\hline Activities & 8-h shift (h:min:s) & Categories \\
\hline Communication with a resident & & \\
Pure & $0: 32: 01$ & Oral communication \\
Concurrent & $1: 28: 53$ & \\
Total & $2: 00: 54$ & \\
Direct care activities that occur concurrently with communication with a resident & & \\
Routine hygiene (e.g. daily shower or wash) & $0: 10: 01$ & Personal hygiene \\
Dressing a resident & $0: 09: 35$ & Personal hygiene \\
Assisting a resident with transfer to and from a bed, a chair, etc. & $0: 08: 56$ & Mobility \\
Transferring a resident to or from the dining room or boardroom & $0: 08: 06$ & Mobility \\
Toileting (prompted by a resident) & $0: 05: 06$ & Toileting \\
Pad change & $0: 02: 55$ & Continence \\
Assisting a resident with eating and drinking (including feeding systems) & $0: 02: 54$ & Nutrition \\
Resident mobility; passive and active exercises; turning a resident in bed & $0: 02: 05$ & Mobility \\
Assisting a resident with food (e.g. cutting up, uncovering or delivery of food) & $0: 01: 57$ & Nutrition \\
Toileting (prompted by a personal care worker) & $0: 01: 07$ & Toileting \\
Total & $0: 54: 00$ & \\
\hline
\end{tabular}

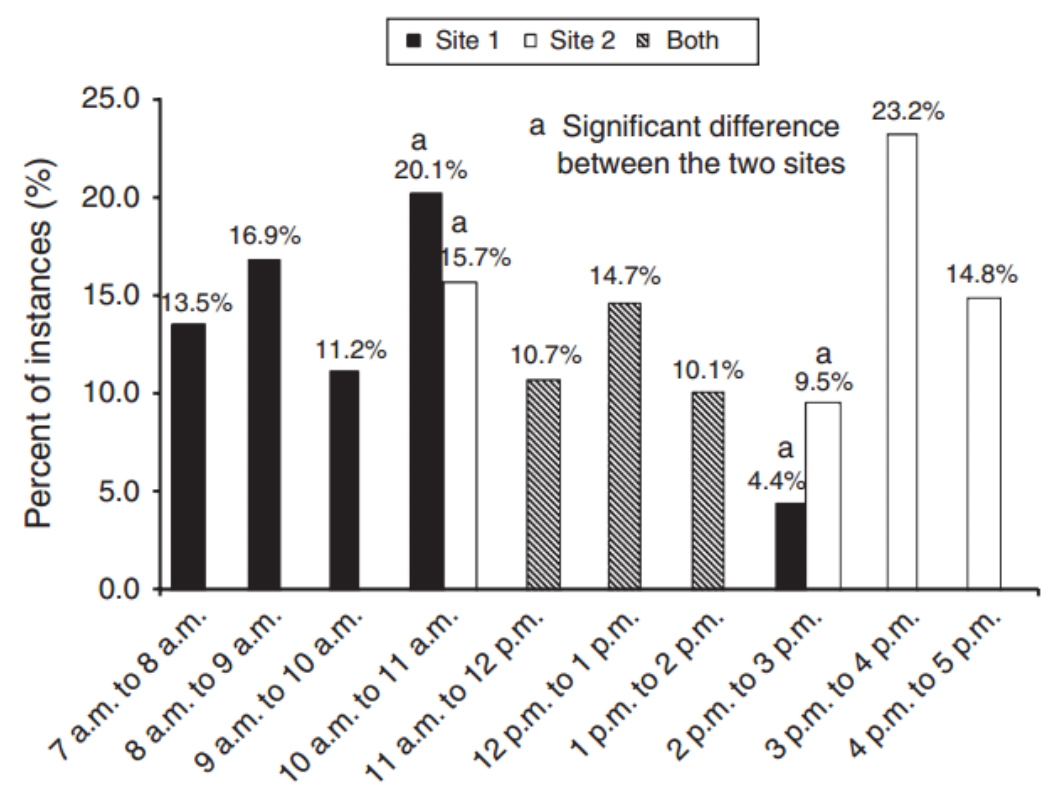

Hour of the day

Fig. 1. Percentage of instances of direct care distributed in different hours over the observation periods at Site 1 and Site 2. 


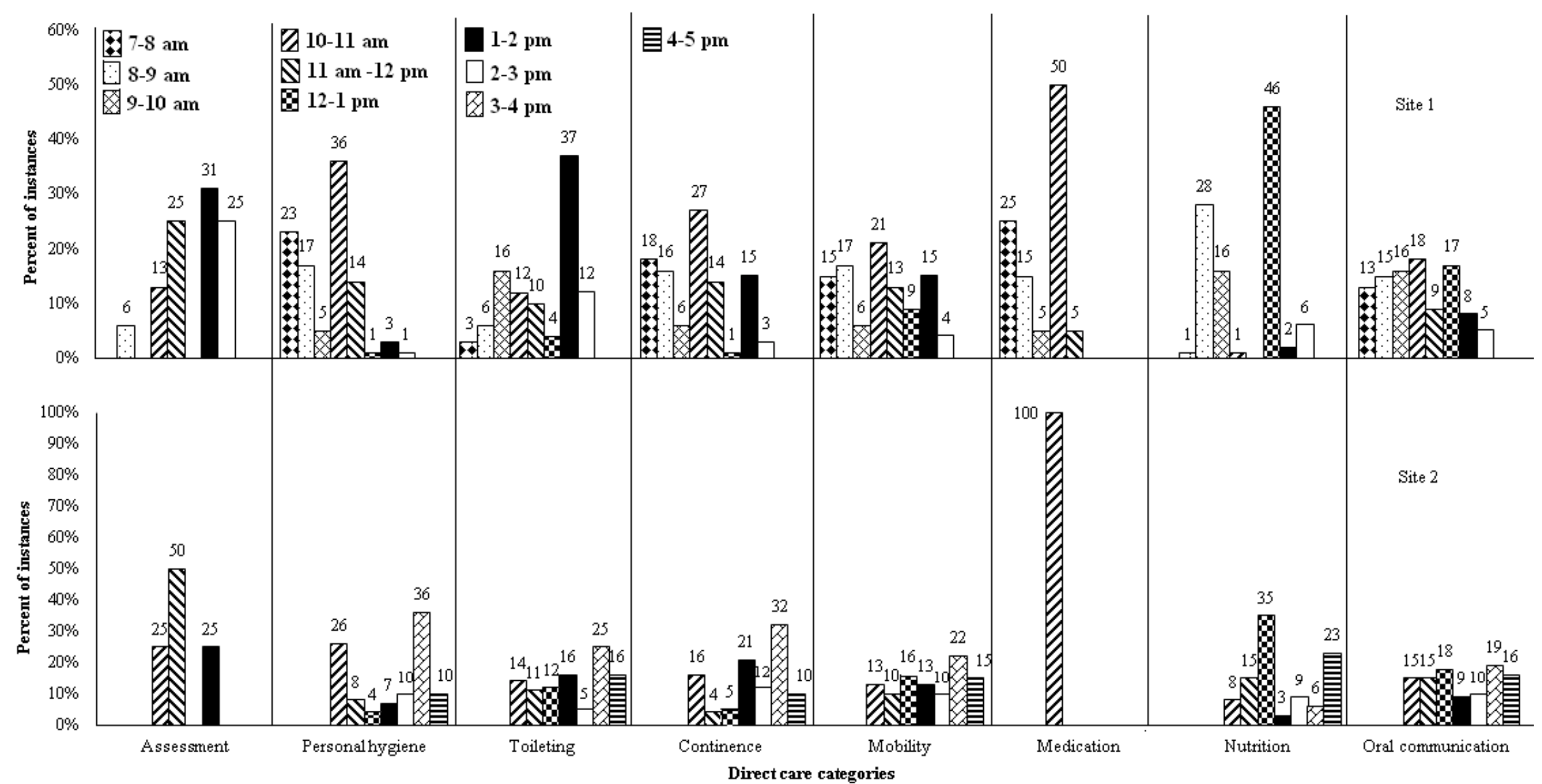

Fig. 2. Percentage of instances of each category of activity distributed in different hours over the observation periods (values at the top of each column are the percentage) 